\title{
Combining QED and QCD transverse-momentum resummation for $Z$ boson production at hadron colliders
}

\author{
Leandro Cieri, ${ }^{a}$ Giancarlo Ferrera ${ }^{b}$ and Germán F.R. Sborlini ${ }^{b}$ \\ ${ }^{a}$ INFN, Sezione di Milano-Bicocca, \\ Piazza della Scienza 3, I-20126 Milan, Italy \\ ${ }^{b}$ Tif Lab, Dipartimento di Fisica, Università di Milano and INFN, Sezione di Milano, \\ Via Celoria 16, I-20133 Milan, Italy \\ E-mail: leandro.cieri@mib.infn.it, giancarlo.ferrera@mi.infn.it, \\ german.sborlini@unimi.it
}

ABSTRACT: We consider the transverse-momentum $\left(q_{T}\right)$ distribution of $Z$ bosons produced in hadronic collisions. At small values of $q_{T}$, we perform the analytic resummation of the logarithmically enhanced QED contributions up to next-to-leading logarithmic accuracy, including the mixed QCD-QED contributions at leading logarithmic accuracy. Resummed results are consistently matched with the next-to-leading fixed-order results (i.e. $\mathcal{O}\left(\alpha^{2}\right)$ ) at small, intermediate and large values of $q_{T}$. We combine the QED corrections with the known QCD results at next-to-next-to-leading order $\left(\mathcal{O}\left(\alpha_{S}^{2}\right)\right)$ and next-to-next-to-leading logarithmic accuracy. We show numerical results at LHC and Tevatron energies, studying the impact of the QED corrections and providing an estimate of the corresponding perturbative uncertainty. Our analytic results for the combined QED and QCD resummation, obtained through an extension of the $q_{T}$ resummation formalism in QCD, are valid for the production of generic neutral and colourless high-mass systems in hadronic collision.

KeYwords: Resummation, Perturbative QCD, Precision QED

ARXIV EPRINT: 1805.11948 


\section{Contents}

1 Introduction 1

2 Combined QED and QCD transverse-momentum resummation 3

$\begin{array}{lll}3 & \text { Numerical results } & 7\end{array}$

4 Summary 11

\section{Introduction}

The production of vector bosons ( $W$ and $Z / \gamma^{*}$ ), through the Drell-Yan (DY) mechanism [1], is one of the most prominent hard-scattering processes at high-energy hadron colliders, such as the Tevatron and the LHC. In particular, it provides strong tests of the Standard Model (SM) and of QCD, it is important for precise measurements of the mass and the width of the $W$ boson and for the extraction of the electroweak (EW) mixing angle, it gives stringent information on the parton densities functions of the colliding hadrons, and it represents an important background to other processes and to various possible signals from physics beyond the SM.

Owing to the above reasons, it is of primary importance to provide accurate theoretical predictions for vector-boson production cross sections and related kinematical distributions. This task requires detailed computations of the higher-order radiative corrections in QCD $[2,3]-[11]$ and in the EW theory [12-16]-[27].

Among the various kinematical distributions, the transverse-momentum $\left(q_{T}\right)$ spectrum of the vector boson has a particularly high relevance. A good understanding of the $Z$ boson $q_{T}$ distribution gives important information on the $W$ boson production mechanism. In turn, an accurate theoretical prediction of the $q_{T}$ distribution of the $W$ boson, in the intermediate and small $q_{T}$ region, is crucial for a precise measurement of the $W$ mass [28-30]. In order to fully exploit the high precision of the LHC measurements, corresponding theoretical predictions with increasing accuracy are therefore essential.

In the large- $q_{T}$ region, where the transverse momentum is of the order of the vector boson mass $M$, fixed-order perturbative calculations are theoretically justified [31-34]. However the bulk of the vector boson cross section lies in the small- $q_{T}$ region $\left(q_{T} \ll M\right)$ where the reliability of the fixed-order expansion is spoiled by the presence of large logarithmic corrections of the type $\ln \left(M^{2} / q_{T}^{2}\right)$ due to the initial state radiation of soft and/or collinear partons.

In order to obtain reliable predictions, the enhanced-logarithmic terms have to be evaluated and systematically resummed to all orders in perturbation theory. The formalism 
to resum these large logarithmic corrections in $\mathrm{QCD}$ has been developed from the late seventies [35-38]-[49] starting from results settled for QED [50].

While the resummation of the large logarithmic corrections in QCD in the small $q_{T}$ region is mandatory, the impact of the large logarithmic QED corrections from the initial states is expected to be substantial only at extremely small values of $q_{T}$ due to the relative smallness of the electromagnetic coupling $\alpha$ with respect to the strong coupling $\alpha_{S}$.

Nonetheless all-order resummation of QED emissions can have non-negligible impact on pure QCD resummed results. Moreover since the first-order QCD contribution at finite value of $q_{T}$ is an $\mathcal{O}\left(\alpha_{S}\right)$ correction relative to the Born-level result, the QED contribution corresponds to an $\mathcal{O}\left(\alpha / \alpha_{S}\right)$ correction to the QCD result. Given the present accuracy of the theoretical predictions [51-53] and the high-precision experimental LHC data, it is therefore important to quantify in a consistent way the impact of QED contributions for the $q_{T}$ distribution of vector bosons.

In this paper we extend the QCD formalism of refs. [46, 47] in order to combine QCD and QED transverse momentum resummation. In particular we explicitly consider the case of $Z$ production at the Tevatron and the LHC by performing QED and QCD resummation up to next-to-leading logarithmic (NLL) and at next-to-next-to-leading logarithmic (NNLL) accuracy, respectively. We also include the resummation of the mixed QCD-QED corrections at leading logarithmic accuracy. In order to achieve a uniform theoretical accuracy for the entire range of transverse momenta, our resummed calculation has been consistently matched at small and intermediate-large values of $q_{T}$ with the fixed-order corrections at next-to-leading order (NLO) in QED (i.e. $\mathcal{O}\left(\alpha^{2}\right)$ ) and next-to-next-to-leading order (NNLO) in QCD (i.e. $\left.\mathcal{O}\left(\alpha_{S}^{2}\right)\right)$. Owing to the unitarity constraint of the resummation formalism, the integral over $q_{T}$ of our predictions with QED effects reproduce the corresponding NLO QED corrections to the total cross section, which have been obtained taking the abelian limit of the corresponding QCD result. ${ }^{1}$

We show that the QED corrections have a non negligible impact on the $q_{T}$ spectrum of the $Z$ boson. In particular the inclusion of the NLL+NLO QED corrections reduces the effect of renormalization scale ambiguity of the QED coupling $\alpha$ by consistently including running coupling effects.

The paper is organized as follows. In section 2 we present the combined QED and QCD resummation formalism, which is valid for a generic process involving a colorless and neutral final state (namely, $Z / \gamma^{*}, Z Z, \gamma \gamma$, Higgs boson(s), etc.), providing the explicit expression for the universal coefficients necessary to include QED resummation up to NLL accuracy. In section 3 we show illustrative numerical results for $Z$ boson production at Tevatron and LHC energies. In particular, we present combined resummation results up to NLL+NLO in QED and NNLL+NNLO in QCD, quantifying the impact of QED effects with respect to the corresponding NNLL+NNLO QCD results. We then perform a study of the scale dependence of the QED corrections in order to estimate the corresponding perturbative uncertainty. In section 4 we summarize our results.

\footnotetext{
${ }^{1}$ Through a similar abelianization procedure the total cross section for on-shell $Z$ production up to $\mathrm{NNLO}$ in QED and in QCD $\times$ QED have been recently obtained in ref. [54].
} 


\section{Combined QED and QCD transverse-momentum resummation}

In this section we extend the QCD transverse-momentum resummation formalism of refs. [46, 47] in order to consistently include the resummation of QED perturbative logarithmic corrections in the generic case of the production of high-mass systems composed by colourless and neutral particles in hadronic collision. In particular we highlight the differences that arise when considering the inclusion of the QED emissions, by considering the specific case of the hadroproduction of an on-shell $Z$ boson: $h_{1}+h_{2} \rightarrow F+X,(F=Z)$.

We start our presentation of combined QCD and QED transverse-momentum resummation by briefly recalling the main features of $q_{T}$ resummation for QCD [46, 47]. According to the QCD factorization theorem, the transverse-momentum differential cross section for this process can be written as

$$
\begin{aligned}
& \frac{d \sigma_{h_{1} h_{2} \rightarrow F}}{d q_{T}^{2}}\left(q_{T}, M, s\right) \\
& =\sum_{a_{1}, a_{2}} \int_{0}^{1} d x_{1} \int_{0}^{1} d x_{2} f_{a_{1} / h_{1}}\left(x_{1}, \mu_{F}^{2}\right) f_{a_{2} / h_{2}}\left(x_{2}, \mu_{F}^{2}\right) \frac{d \hat{\sigma}_{a_{1} a_{2} \rightarrow F}}{d q_{T}^{2}}\left(q_{T}, M, \hat{s} ; \mu_{F}^{2}\right),
\end{aligned}
$$

where $f_{a / h}\left(x, \mu_{F}^{2}\right)(a=q, \bar{q}, g)$ are the parton densities of the hadron $h$ at the factorization scale $\mu_{F}, d \hat{\sigma}_{a_{1} a_{2} \rightarrow F}$ are the partonic cross sections and $s\left(\hat{s}=x_{1} x_{2} s\right)$ is the hadronic (partonic) centre-of-mass energy.

The partonic cross sections $d \hat{\sigma} / d q_{T}^{2}$ in eq. (2.1) can be decomposed as follows: $d \hat{\sigma}=d \hat{\sigma}^{\text {(res. })}+d \hat{\sigma}^{\text {(fin.) }}$, where the 'resummed' component $\left(d \hat{\sigma}^{\text {(res. })}\right)$ contains all the logarithmically enhanced contributions at small $q_{T}$ proportional to $\delta\left(q_{T}^{2}\right)$ or to $1 / q_{T}^{2} \ln ^{m}\left(M^{2} / q_{T}^{2}\right)$ and the 'finite' component $\left(d \hat{\sigma}^{(\text {fin. })}\right)$ is free of such contributions.

The resummation procedure is carried out in the impact-parameter $(b)$ space conjugated to $q_{T}$. The resummed component is then obtained by performing the inverse Bessel transformation with respect to $b$ :

$$
\frac{d \hat{\sigma}_{a_{1} a_{2} \rightarrow F}^{\text {(res.) }}}{d q_{T}^{2}}\left(q_{T}, M, \hat{s} ; \mu_{F}\right)=\frac{M^{2}}{\hat{s}} \int_{0}^{\infty} d b \frac{b}{2} J_{0}\left(b q_{T}\right) \mathcal{W}_{a_{1} a_{2}}^{F}\left(b, M, \hat{s} ; \mu_{F}\right)
$$

where $J_{0}(x)$ is the 0th-order Bessel function. The resummation structure of $\mathcal{W}_{a_{1} a_{2}}^{F}$ can be organized in an exponential form by considering the Mellin $N$-moments $\mathcal{W}_{a_{1} a_{2}, N}^{F},{ }^{2}$ with respect to $z=M^{2} / \hat{s}$ at fixed $M$. In the simplified flavour-diagonal case $\left(a_{1} a_{2}=c \bar{c}\right)$ it reads:

$$
\begin{aligned}
\mathcal{W}_{N}^{F}\left(b, M ; \mu_{F}\right)= & \hat{\sigma}_{F}^{(0)}(M) \mathcal{H}_{N}^{F}\left(\alpha_{S} ; M^{2} / \mu_{R}^{2}, M^{2} / \mu_{F}^{2}, M^{2} / Q^{2}\right) \\
& \times \exp \left\{\mathcal{G}_{N}\left(\alpha_{S}, L ; M^{2} / \mu_{R}^{2}, M^{2} / Q^{2}\right)\right\}
\end{aligned}
$$

where $\hat{\sigma}_{F}^{(0)}$ is the lowest-order partonic cross section, $L=\log \left(Q^{2} b^{2} / b_{0}^{2}+1\right)$ is the logarithmic expansion parameter, $Q$ is the resummation scale [47] and $b_{0}=2 e^{-\gamma_{E}}$ (with $\gamma_{E}=0.5772 \ldots$ the Euler-Mascheroni constant). In eq. (2.3) we explicitly introduced the renormalization scale $\mu_{R}$ and the perturbative QCD coupling $\alpha_{S}=\alpha_{S}\left(\mu_{R}^{2}\right)$.

\footnotetext{
${ }^{2}$ The $N$-moments of a generic function $h(z)$ are defined as $h_{N}=\int_{0}^{1} d z z^{N-1} h(z)$.
} 
The universal form factor $\exp \left\{\mathcal{G}_{N}\right\}$ includes (and resums to all orders) the large logarithmic terms $\alpha_{S}^{n} L^{m}(1 \leq m \leq 2 n)$. It can be systematically expanded in powers of $\alpha_{S}$ as follows:

$$
\mathcal{G}_{N}\left(\alpha_{S}, L\right)=L g^{(1)}\left(\alpha_{S} L\right)+g_{N}^{(2)}\left(\alpha_{S} L\right)+\frac{\alpha_{S}}{\pi} g_{N}^{(3)}\left(\alpha_{S} L\right)+\sum_{n=4}^{\infty}\left(\frac{\alpha_{S}}{\pi}\right)^{n-2} g_{N}^{(n)}\left(\alpha_{S} L\right),
$$

where the term $L g^{(1)}$ collects the leading logarithmic (LL) contributions, the function $g_{N}^{(2)}$ controls the NLL contributions, $g_{N}^{(3)}$ includes the NNLL terms and so forth.

All the perturbative terms that behave as constants in the limit $b \rightarrow \infty$ are included in the process dependent hard-collinear function $\mathcal{H}_{N}^{F}$ which has a customary perturbative expansion:

$$
\mathcal{H}_{N}^{F}\left(\alpha_{S}\right)=1+\frac{\alpha_{S}}{\pi} \mathcal{H}_{N}^{F(1)}+\left(\frac{\alpha_{S}}{\pi}\right)^{2} \mathcal{H}_{N}^{F(2)}+\sum_{n=3}^{\infty}\left(\frac{\alpha_{S}}{\pi}\right)^{n} \mathcal{H}_{N}^{F(n)}
$$

We now discuss how to extend the QCD resummation formalism in order to include and consistently resum the large logarithmic QED corrections of the type $\alpha^{n} L^{m}(1 \leq m \leq 2 n)$. We start form eq. (2.1) and we consider also the inclusion of the photon parton density $f_{\gamma / h}\left(x, \mu_{F}^{2}\right)$. The eq. (2.3) have then to be replaced by the following generalized expressions: ${ }^{3}$

$$
\begin{aligned}
\mathcal{W}_{N}^{\prime F}\left(b, M ; \mu_{F}\right)= & \hat{\sigma}_{F}^{(0)}(M) \mathcal{H}_{N}^{\prime F}\left(\alpha_{S}, \alpha ; M^{2} / \mu_{R}^{2}, M^{2} / \mu_{F}^{2}, M^{2} / Q^{2}\right) \\
& \times \exp \left\{\mathcal{G}_{N}^{\prime}\left(\alpha_{S}, \alpha, L ; M^{2} / \mu_{R}^{2}, M^{2} / Q^{2}\right)\right\}
\end{aligned}
$$

where the form factor $\mathcal{G}_{N}^{\prime}\left(\alpha_{S}, \alpha, L\right)$ in eq. (2.6) has a double perturbative expansion in powers of $\alpha_{S}$ and $\alpha$

$$
\begin{aligned}
\mathcal{G}_{N}^{\prime}\left(\alpha_{S}, \alpha, L\right)= & \mathcal{G}_{N}\left(\alpha_{S}, L\right)+L g^{\prime(1)}(\alpha L)+g_{N}^{\prime(2)}(\alpha L)+\sum_{n=3}^{\infty}\left(\frac{\alpha}{\pi}\right)^{n-2} g_{N}^{\prime(n)}(\alpha L) \\
& +g^{\prime(1,1)}\left(\alpha_{S} L, \alpha L\right)+\sum_{\substack{n, m=1 \\
n+m \neq 2}}^{\infty}\left(\frac{\alpha_{S}}{\pi}\right)^{n-1}\left(\frac{\alpha}{\pi}\right)^{m-1} g_{N}^{\prime(n, m)}\left(\alpha_{S} L, \alpha L\right)
\end{aligned}
$$

where $\alpha=\alpha\left(\mu_{R}\right)$ is the electromagnetic coupling evaluated at the renormalization scale $\mu_{R}$. The term $L g^{(1)}$ collects the LL contributions in QED, the function $g_{N}^{\prime(2)}$ controls the NLL QED and so forth, while the terms $g^{\prime(1,1)}\left(\alpha_{S} L, \alpha L\right)$ and $g_{N}^{\prime(n, m)}\left(\alpha_{S} L, \alpha L\right)$ include respectively the leading and subleading mixed QCD-QED corrections. The extension of the eq. (2.5) has an analogous double perturbative expansion in powers of $\alpha_{S}$ and $\alpha$ :

$$
\begin{aligned}
\mathcal{H}_{N}^{\prime F}\left(\alpha_{S}, \alpha\right)= & \mathcal{H}_{N}^{F}\left(\alpha_{S}\right)+\frac{\alpha}{\pi} \mathcal{H}_{N}^{\prime F(1)}+\sum_{n=2}^{\infty}\left(\frac{\alpha}{\pi}\right)^{n} \mathcal{H}_{N}^{\prime F(n)} \\
& +\sum_{n, m=1}^{\infty}\left(\frac{\alpha_{S}}{\pi}\right)^{n}\left(\frac{\alpha}{\pi}\right)^{m} \mathcal{H}_{N}^{\prime F(n, m)}
\end{aligned}
$$

\footnotetext{
${ }^{3}$ In order to simplify the expressions in this section we do not explicitly introduce an additional dependence on perturbative scales in QED (i.e. we fix them to be equal to the corresponding QCD ones).
} 
where the pure QED corrections are controlled by the coefficients $\mathcal{H}_{N}^{\prime F(n)}$ while the mixed QCD-QED ones are contained in the coefficients $\mathcal{H}_{N}^{\prime F(n, m)}$.

The LL and NLL functions $g^{\prime(1)}$ and $g_{N}^{(2)}$ in eq. (2.7) have the same functional form of the corresponding QCD ones

$$
\begin{aligned}
g^{\prime(1)}(\alpha L)= & \frac{A_{q}^{\prime(1)}}{\beta_{0}^{\prime}} \frac{\lambda^{\prime}+\ln \left(1-\lambda^{\prime}\right)}{\lambda^{\prime}}, \\
g_{N}^{\prime(2)}(\alpha L)= & \frac{\widetilde{B}_{q, N}^{\prime(1)}}{\beta_{0}^{\prime}} \ln \left(1-\lambda^{\prime}\right)-\frac{A_{q}^{\prime(2)}}{\beta_{0}^{\prime 2}}\left(\frac{\lambda^{\prime}}{1-\lambda^{\prime}}+\ln \left(1-\lambda^{\prime}\right)\right) \\
& +\frac{A_{q}^{\prime(1)} \beta_{1}^{\prime}}{\beta_{0}^{\prime 3}}\left(\frac{1}{2} \ln ^{2}\left(1-\lambda^{\prime}\right)+\frac{\ln \left(1-\lambda^{\prime}\right)}{1-\lambda^{\prime}}+\frac{\lambda^{\prime}}{1-\lambda^{\prime}}\right),
\end{aligned}
$$

while the novel function $g^{\prime(1,1)}\left(\alpha_{S} L, \alpha L\right)$, which controls the mixed QCD-QED correction at leading logarithmic accuracy, reads

$$
g^{\prime(1,1)}\left(\alpha_{S} L, \alpha L\right)=\frac{A_{q}^{(1)} \beta_{0,1}}{\beta_{0}^{2} \beta_{0}^{\prime}} h\left(\lambda, \lambda^{\prime}\right)+\frac{A_{q}^{\prime(1)} \beta_{0,1}^{\prime}}{\beta_{0}^{\prime 2} \beta_{0}} h\left(\lambda^{\prime}, \lambda\right),
$$

with

$$
\begin{aligned}
h\left(\lambda, \lambda^{\prime}\right)= & -\frac{\lambda^{\prime}}{\lambda-\lambda^{\prime}} \ln (1-\lambda)+\ln \left(1-\lambda^{\prime}\right)\left[\frac{\lambda\left(1-\lambda^{\prime}\right)}{(1-\lambda)\left(\lambda-\lambda^{\prime}\right)}+\ln \left(\frac{-\lambda^{\prime}(1-\lambda)}{\lambda-\lambda^{\prime}}\right)\right] \\
& -\operatorname{Li}_{2}\left(\frac{\lambda}{\lambda-\lambda^{\prime}}\right)+\operatorname{Li}_{2}\left(\frac{\lambda\left(1-\lambda^{\prime}\right)}{\lambda-\lambda^{\prime}}\right)
\end{aligned}
$$

where $\lambda=\frac{1}{\pi} \beta_{0} \alpha_{S} L, \lambda^{\prime}=\frac{1}{\pi} \beta_{0}^{\prime} \alpha L$, and $\beta_{0}, \beta_{0}^{\prime}, \beta_{1}^{\prime}, \beta_{0,1}, \beta_{0,1}^{\prime}$ are the coefficients of the QCD and QED $\beta$ functions:

$$
\begin{aligned}
& \frac{d \ln \alpha_{S}\left(\mu^{2}\right)}{d \ln \mu^{2}}=\beta\left(\alpha_{S}\left(\mu^{2}\right), \alpha\left(\mu^{2}\right)\right)=-\sum_{n=0}^{\infty} \beta_{n}\left(\frac{\alpha_{S}}{\pi}\right)^{n+1}-\sum_{n, m+1=0}^{\infty} \beta_{n, m}\left(\frac{\alpha_{S}}{\pi}\right)^{n+1}\left(\frac{\alpha}{\pi}\right)^{m} \\
& \frac{d \ln \alpha\left(\mu^{2}\right)}{d \ln \mu^{2}}=\beta^{\prime}\left(\alpha\left(\mu^{2}\right), \alpha_{S}\left(\mu^{2}\right)\right)=-\sum_{n=0}^{\infty} \beta_{n}^{\prime}\left(\frac{\alpha}{\pi}\right)^{n+1}-\sum_{n, m+1=0}^{\infty} \beta_{n, m}^{\prime}\left(\frac{\alpha}{\pi}\right)^{n+1}\left(\frac{\alpha_{S}}{\pi}\right)^{m}
\end{aligned}
$$

The function $g^{\prime(1,1)}$ in eqs. $(2.11),(2.12)$ has been obtained by plain integration, starting from the integral representation (see e.g. eq. (19) of ref. [47])) of the form factor in eq. (2.7). The mixed QCD-QED terms included in eqs. (2.11), (2.12) originate from the QED evolution of the QCD coupling and from the QCD evolution of the QED coupling, respectively in the pure QCD and QED form factors. Indeed in eqs. (2.13), (2.14) we have consistently included the mixed QCD-QED contributions to the running of the QCD and QED couplings through the coefficient $\beta_{n, m}$ and $\beta_{n, m}^{\prime}$. The explicit expressions of the 
coefficients $\beta_{0}, \beta_{0}^{\prime}, \beta_{1}^{\prime}, \beta_{0,1}, \beta_{0,1}^{\prime}$ are:

$$
\begin{array}{lll}
\beta_{0}=\frac{1}{12}\left(11 C_{A}-2 n_{f}\right), & \beta_{0,1}=-\frac{N_{q}^{(2)}}{8}, & \\
\beta_{0}^{\prime}=-\frac{N^{(2)}}{3}, & \beta_{1}^{\prime}=-\frac{N^{(4)}}{4}, & \beta_{0,1}^{\prime}=-\frac{C_{F} C_{A} N_{q}^{(2)}}{4},
\end{array}
$$

where we have defined

$$
\begin{aligned}
& N^{(n)}=N_{c} \sum_{q=1}^{n_{f}} e_{q}^{n}+\sum_{l=1}^{n_{l}} e_{l}^{n}, \\
& N_{q}^{(n)}=\sum_{q=1}^{n_{f}} e_{q}^{n},
\end{aligned}
$$

with $N_{c}=3$ the number of colours, $C_{A}=N_{c}, C_{F}=\left(N_{c}^{2}-1\right) /\left(2 N_{c}\right), n_{f}\left(n_{l}\right)$ the number of quark (lepton) flavours and $e_{q}\left(e_{l}\right)$ the quark (lepton) electric charges $\left(e_{q}=2 / 3\right.$ for up-type quarks, $e_{q}=-1 / 3$ for down-type quarks, $e_{l}=1$ for leptons).

The coefficients $A_{q}^{\prime(1)}$ and $A_{q}^{\prime(2)}$, which have been obtained from the corresponding coefficients in QCD [42-44,55] through an Abelianization algorithm [56, 57], read

$$
A_{q}^{\prime(1)}=e_{q}^{2}, \quad A_{q}^{\prime(2)}=-\frac{5}{9} e_{q}^{2} N^{(2)},
$$

while the coefficient $\widetilde{B}_{q, N}^{\prime(1)}$ is

$$
\widetilde{B}_{q, N}^{\prime(1)}=B_{q}^{\prime(1)}+2 \gamma_{q q, N}^{\prime(1)}
$$

with

$$
\begin{aligned}
B_{q}^{\prime(1)} & =-\frac{3}{2} e_{q}^{2} \\
\gamma_{q q, N}^{\prime(1)} & =e_{q}^{2}\left(\frac{3}{4}+\frac{1}{2 N(N+1)}-\gamma_{E}-\psi_{0}(N+1)\right), \\
\gamma_{q \gamma, N}^{\prime(1)} & =\frac{3}{2} e_{q}^{2} \frac{N^{2}+N+2}{N(N+1)(N+2)},
\end{aligned}
$$

where $\psi_{0}(N)$ is the digamma function and $\gamma_{a b, N}^{\prime(1)}$ are the leading-order (LO) anomalous dimensions in QED. ${ }^{4}$

The LL mixed QCD-QED corrections are included in the $g^{\prime(1,1)}\left(\alpha_{S} L, \alpha L\right)$ function which depends on the coefficients $A_{q}^{(1)}=C_{F}, A_{q}^{\prime(1)}, \beta_{0}, \beta_{0}^{\prime}, \beta_{0,1}, \beta_{0,1}^{\prime}$. We note that these logarithmic terms do not contribute to the $\mathcal{O}\left(\alpha \alpha_{S}\right)$ fixed order corrections and their dominant contribution is of the order $\alpha \alpha_{S}^{2} L^{3}$.

\footnotetext{
${ }^{4}$ The QED anomalous dimension $\gamma_{q \gamma, N}^{\prime(1)}$ is required at the NLL in the general multiflavour case (see appendix A of ref. [47]).
} 
In order to reach the complete NLL+NLO accuracy for QED, the functions $\mathcal{H}_{a_{1} a_{2}, N}^{\prime F(1)}$ and the finite component of the partonic cross sections $d \hat{\sigma}_{a_{1} a_{2}}^{(\text {fin.) }}$ at the first non-trivial order are needed. The hard-collinear functions $\mathcal{H}_{a_{1} a_{2}, N}^{\prime F(1)}$ read

$$
\begin{aligned}
& \mathcal{H}_{q \bar{q} \leftarrow q \bar{q}, N}^{\prime F(1)}=\frac{e_{q}^{2}}{2}\left(\frac{2}{N(N+1)}-8+\pi^{2}\right), \\
& \mathcal{H}_{q \bar{q} \leftarrow \gamma q, N}^{\prime F(1)}=\mathcal{H}_{q \bar{q} \leftarrow q \gamma, N}^{\prime F(1)}=\frac{3 e_{q}^{2}}{(N+1)(N+2)}, \\
& \mathcal{H}_{q \bar{q} \leftarrow \gamma \gamma, N}^{\prime F(1)}=\mathcal{H}_{q \bar{q} \leftarrow q q, N}^{\prime F(1)}=\mathcal{H}_{q \bar{q} \leftarrow \bar{q} \bar{q}, N}^{\prime F(1)}=0 .
\end{aligned}
$$

The explicit perturbative scale dependence of the formulae in eqs. (2.9), (2.10), (2.24) is the same as the corresponding one in QCD (see eqs. (22), (23), (46) of ref. [47]).

\section{Numerical results}

In this section we present selected numerical results, by explicitly considering the transverse-momentum distribution of on-shell $Z$ bosons in hadronic collisions. We start our analysis from the resummed results in QCD at NNLL+NNLO as implemented in the DYqT numerical code $[58,59]$ and we include the QED corrections up to NLL+NLO.

As for the electroweak couplings, we use the following input parameters: $\alpha\left(m_{Z}^{2}\right)=$ $1 / 127.95, \sin ^{2} \theta_{W}=0.23129$ and $m_{Z}=91.1876 \mathrm{GeV}$ [60]. The electromagnetic coupling $\alpha$ is evaluated at 1-loop and 2-loops respectively at LO and NLO in QED. The hadronic cross section is computed using the NNPDF3.1QED parton distribution function (PDF) set at NNLO in QCD [61, 62], which includes the photon PDF as determined within the LUX method [63, 64] as well as the LO QED effects in the evolution of the parton densities. The strong coupling $\alpha_{S}$ is evaluated at 3-loop order with $\alpha_{S}\left(m_{Z}^{2}\right)=0.118$ within the $\overline{\mathrm{MS}}$ renormalization scheme. We work with $n_{l}=3$ charged leptons and $n_{f}=5$ flavours of light quarks in the massless approximation.

We set the central value of the renormalization, factorization and resummation scales at $\mu_{R}=\mu_{F}=2 Q=m_{Z}$. We provide an estimate of the perturbative uncertainties of the calculation due to missing higher-order QED terms performing the variation of the renormalization $\left(\mu_{R}^{\prime}\right)$ and resummation $\left(Q^{\prime}\right)$ scales associated to the QED contributions. Specifically, the variation of $\mu_{R}^{\prime}$ and $Q^{\prime}$ around their central value can be used to estimate the effects of yet uncalculated QED contributions respectively at fixed order and logarithmic accuracy level. We thus perform an independent variation of $\mu_{R}^{\prime}$ and $Q^{\prime}$ in the range $m_{Z} / 2 \leq\left\{\mu_{R}^{\prime}, 2 Q^{\prime}\right\} \leq 2 m_{Z}$ with the constraint $1 / 2 \leq\left\{\mu_{R}^{\prime} / Q^{\prime}\right\} \leq 2$. In principle it would be possible to consider an independent variation also for the factorization scale $\left(\mu_{F}^{\prime}\right)$ related to the QED emissions. However since the QCD and QED factorization scales are assumed to be equal in the evolution of parton densities [62] we keep the factorization scale fixed at the central value $\mu_{F}^{\prime}=\mu_{F}=m_{Z}$ in our numerical study. The dependence of the resummed QCD predictions from factorization, renormalization and resummation scales has been studied in detail in refs. [58, 59, 65]. 

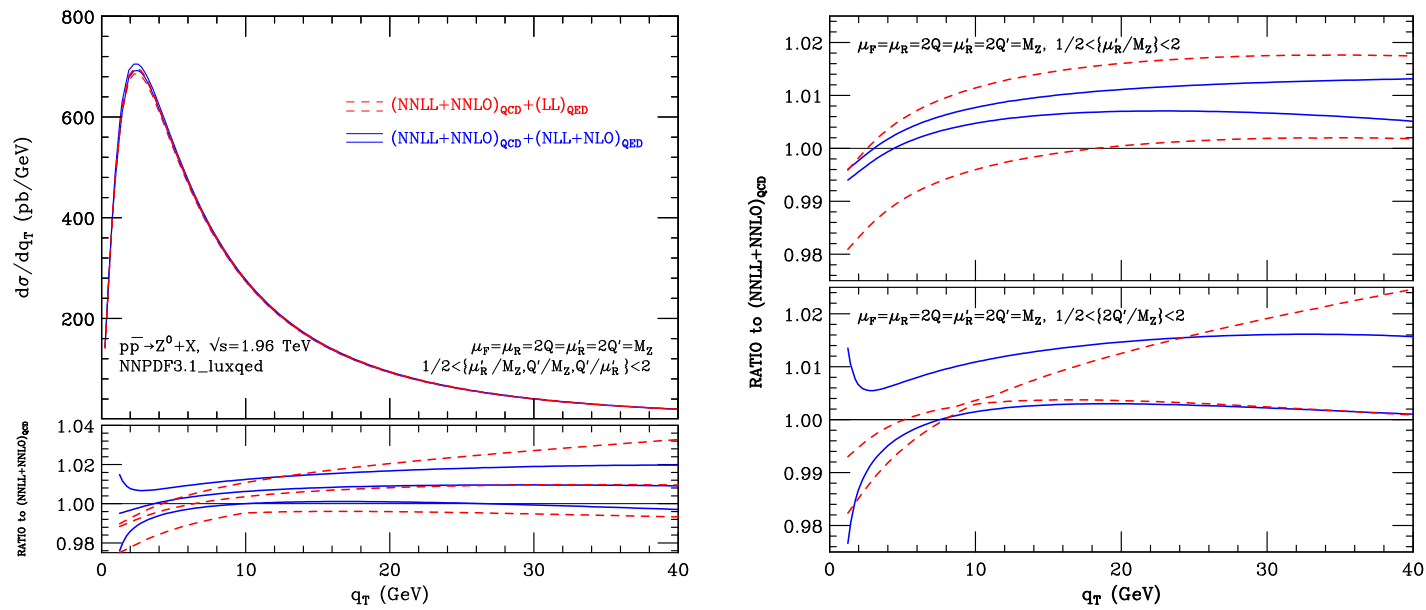

Figure 1. The $q_{T}$ spectrum of $Z$ boson at the Tevatron $(\sqrt{s}=1.96 \mathrm{TeV})$. Left panel: NNLL+NNLO QCD results are combined with the LL (red dashed) and NLL+NLO (blue solid) QED effects together with the corresponding QED uncertainty bands. The bands are obtained by performing the variation of the $\mu_{R}^{\prime}$ and $Q^{\prime}$ scales in QED around their central value as described in the text. The lower panel shows the ratio of the QED scale-dependent results with respect to the standard NNLL+NNLO QCD result at central value of the scales. Right panel: upper (lower) panel shows the ratio of the resummation (renormalization) QED scale-dependent results with respect to the standard NNLL+NNLO QCD result at central value of the scales.

We start considering on-shell $Z$ production in $p \bar{p}$ collisions at Tevatron Run II energies $(\sqrt{s}=1.96 \mathrm{TeV})$. In figure 1 (left panel) we present the NNLL+NNLO QCD results combined with the LL (red dashed) and NLL+NLO (blue solid) QED corrections. The lower panel presents the ratio of our predictions with respect to the standard NNLL+NNLO QCD result at the corresponding central scale.

We observe that the resummation of the QED contributions at LL accuracy for central scale values $\left(\mu_{R}^{\prime}=2 Q^{\prime}=m_{Z}\right)$ has the effect to make the $q_{T}$ spectrum slightly harder. The QED effects at LL are negative in the small $q_{T}$ region $\left(q_{T} \lesssim 6 \mathrm{GeV}\right)$ while they are positive in the $q_{T}$ region $6 \lesssim q_{T} \lesssim 40 \mathrm{GeV}$. The impact of these effects reaches the $\mathcal{O}(1 \%)$ level. We recall that, owing to the unitarity constraint of the resummation formalism that we used [47], the LL QED effects for central values of the scales give vanishing contribution to the total (i.e. $q_{T}$ integrated) cross section: they only affect the shape of the $q_{T}$ distribution by (slightly) shifting part of the cross section to higher values of $q_{T}$. This physical effect to the $q_{T}$ distribution is expected and it is due to the contribution to the $Z$ recoil generated by soft and collinear photon emissions to all orders in QED. The NLL+NLO QED effects at central value of the scales $\mu_{R}^{\prime}=2 Q^{\prime}=m_{Z}$ are of $\mathcal{O}(0.5 \%)$ level and they are positive for the entire $q_{T}$ region considered $\left(q_{T} \lesssim 40 \mathrm{GeV}\right)$. Thanks to the perturbative unitarity of the resummation formalism, the contribution to the integrated $q_{T}$ distribution of the NLL+NLO QED result exactly coincides with the NLO QED correction to the total cross section which is equal to $+0.3 \%$. 
By considering the scale variation band, we observe that the LL QED effects have an uncertainty of around $2 \%$ in the small $q_{T}$ region $\left(q_{T} \lesssim 6 \mathrm{GeV}\right)$ which increases up to $4 \%$ in the intermediate $q_{T}$ region $\left(30 \lesssim q_{T} \lesssim 40 \mathrm{GeV}\right)$. The inclusion of the NLL+NLO QED corrections reduces the scale variation band by roughly a factor 2 . In order to better illustrate the dependence on the QED scales, in figure 1 (right panel) we separately consider $\mu_{R}^{\prime}$ and $Q^{\prime}$ variations. The upper plot corresponds to the variation of $\mu_{R}^{\prime}$ by a factor 2 around the central value and the lower plot corresponds to an analogous variation of $Q^{\prime}$. As expected from the QED running of $\alpha$, the $q_{T}$ distribution decreases (increases) by decreasing (increasing) the value of $\mu_{R}^{\prime}$. The $\mu_{R}^{\prime}$ scale dependence is flat and at the level of $2 \%$ at LL and it decreases to around $0.5-1 \%$ at NLL+NLO. The $Q^{\prime}$ dependence does not affect the integral of the $q_{T}$ distribution which is constrained by perturbative unitarity. The resummation scale dependence at LL is about $1 \%$ for $q_{T} \lesssim 5 \mathrm{GeV}$, it reduces up to $0.1 \%$ for $q_{T} \sim 10 \mathrm{GeV}$ and it increases again up to about $2 \%$ for $q_{T} \sim 30-40 \mathrm{GeV}$. The reduction of the $Q^{\prime}$ dependence in the region $q_{T} \sim 10 \mathrm{GeV}$ is due to the crossing (necessary in order to fulfill unitarity) between the predictions with $Q^{\prime}=m_{Z}$ and $Q^{\prime}=m_{Z} / 4$. This accidental cancellation suggests that the $Q^{\prime}$ variation band at LL might underestimates the perturbative uncertainty of the prediction in the region around $q_{T} \sim 10 \mathrm{GeV}$. In fact at NLL+NLO the $Q^{\prime}$ dependence for $q_{T} \gtrsim 4 \mathrm{GeV}$ is constant and at $\mathcal{O}(1 \%)$ level. Below the peak the $Q^{\prime}$ dependence rapidly increases. We note that in the region $q_{T} \lesssim 3 \mathrm{GeV}$ the $q_{T}$ distribution is steeply falling to zero. We finally observe that at LL the scale variation is mainly driven by $\mu_{R}^{\prime}$ because the Born-level cross section is proportional to $\alpha$, while at NLL+NLO the scale uncertainty is dominated by the $Q^{\prime}$ dependence.

The integral over $q_{T}$ of the predictions with NLL+NLO QED effects are in agreement (for fixed values of renormalization and factorization scales) with the corresponding NLO QED contributions to the total cross section at the $\mathcal{O}(0.1 \%)$ level, ${ }^{5}$ thus checking the numerical accuracy of our implementation.

We now turn to consider on-shell $Z$ production in $p p$ collisions at the LHC energies. In figure 2 (left panel) we present our results for LHC Run I $(\sqrt{s}=8 \mathrm{TeV})$ and in figure 3 (left panel) the results for LHC Run II $(\sqrt{s}=13 \mathrm{TeV})$. The effects of the QED contributions at the LHC are qualitatively similar and quantitatively slightly smaller with respect to the case of the Tevatron.

From the left panels of figures 2 and 3 we observe that the QED effects at LL accuracy, for central value of the scales, give a negative $\mathcal{O}(1 \%)$ contribution in the small $q_{T}$ region $\left(q_{T} \lesssim 8-10 \mathrm{GeV}\right)$ and a positive $\mathcal{O}(0.5 \%)$ contribution in the $q_{T}$ region $10 \lesssim q_{T} \lesssim 40 \mathrm{GeV}$. The NLL+NLO QED effects are at the level of $\mathcal{O}(0.5 \%)$ and positive for the entire $q_{T}$ region considered $\left(q_{T} \lesssim 40 \mathrm{GeV}\right)$. The NLO QED contribution to the total cross section is equal to $+0.3 \%$.

The impact on the $q_{T}$ distributions of the mixed QED-QCD corrections is numerically very small (lower than $0.1 \%$ level) both at the Tevatron and at the LHC, and it has not been separately shown in the plots of figures $1-3$.

\footnotetext{
${ }^{5}$ The NLO QED $\left(\mathcal{O}\left(\alpha^{2}\right)\right)$ corrections to the total cross section and to the finite component $d \hat{\sigma}^{(\text {fin.) }}$ of the $q_{T}$ distribution have been obtained through the Abelianization of the calculations in refs. [2, 3] and in ref. [33] respectively.
} 

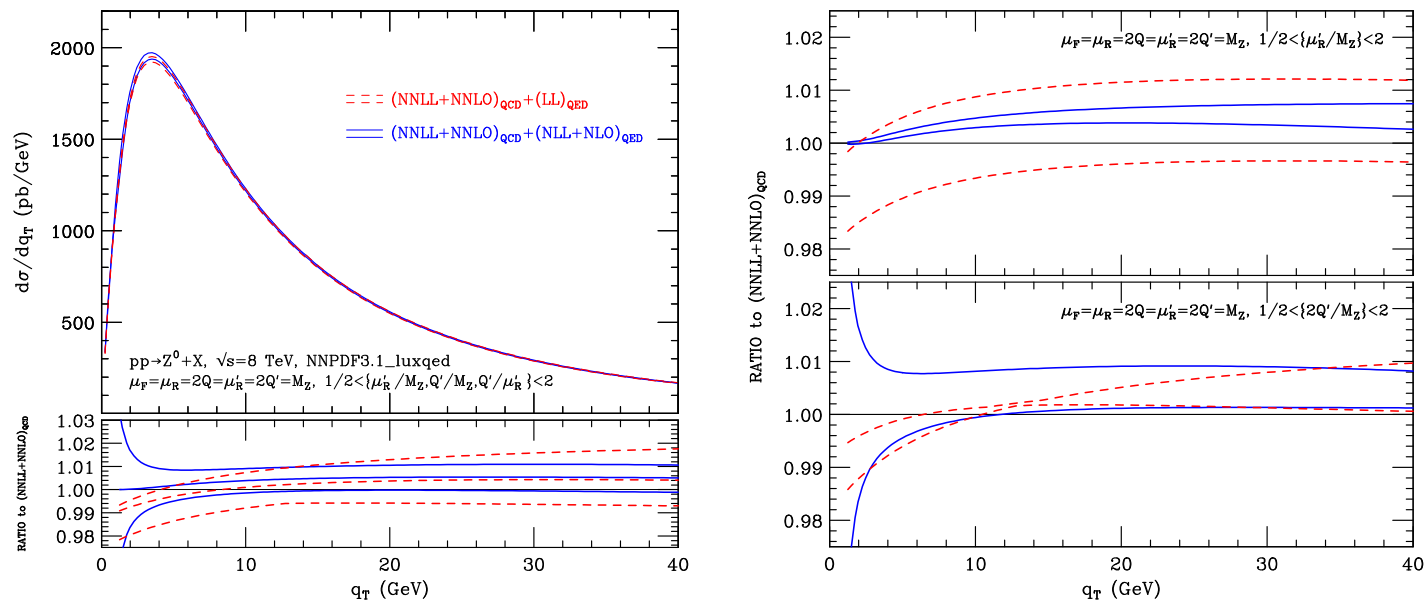

Figure 2. The $q_{T}$ spectrum of $Z$ boson at the LHC $(\sqrt{s}=8 \mathrm{TeV})$. Left panel: NNLL+NNLO QCD results are combined with the LL (red dashed) and NLL+NLO (blue solid) QED effects together with the corresponding QED uncertainty bands. The bands are obtained as in figure 1 .
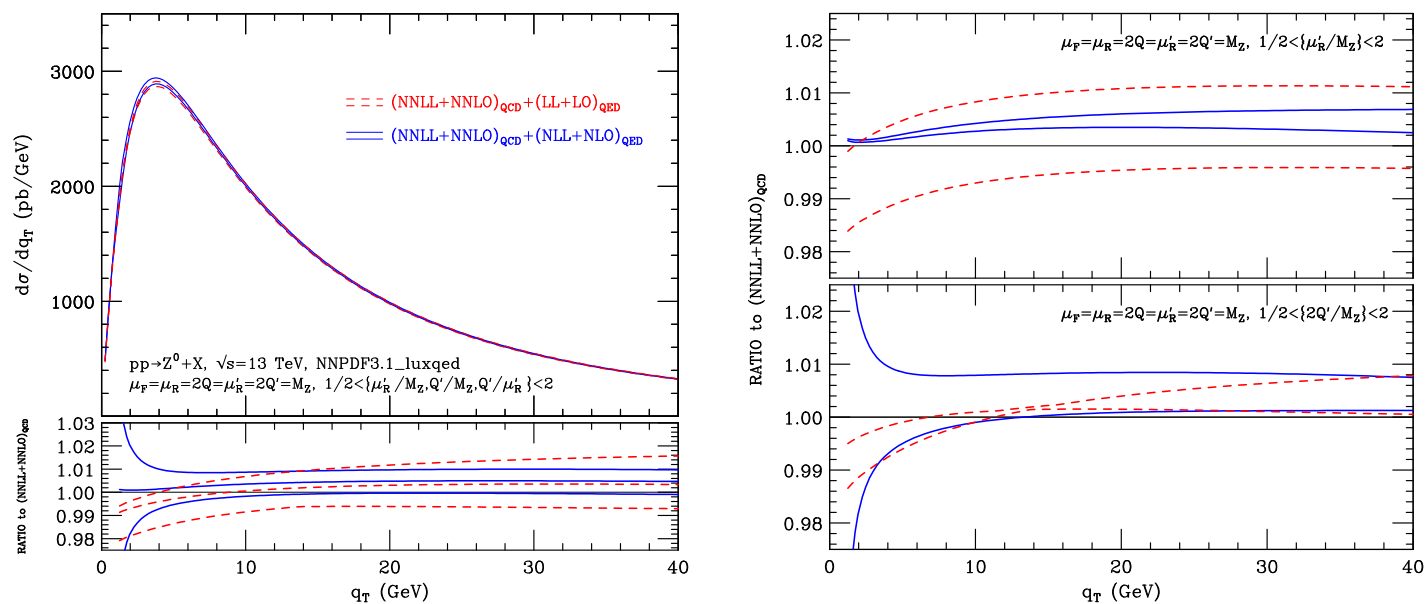

Figure 3. The $q_{T}$ spectrum of $Z$ boson at the LHC $(\sqrt{s}=13 \mathrm{TeV})$. Left panel: NNLL+NNLO QCD results are combined with the LL (red dashed) and NLL+NLO (blue solid) QED effects together with the corresponding QED uncertainty bands. The bands are obtained as in figures 1 and 2 .

By considering the perturbative uncertainty shown in the lower-left panels of figures 2 and 3, we observe that the LL QED effects have an uncertainty of around $2 \%$ in the small $q_{T}$ region $\left(q_{T} \lesssim 10 \mathrm{GeV}\right)$ which increases up to $2.5-3 \%$ in the intermediate $q_{T}$ region $\left(30 \lesssim q_{T} \lesssim 40 \mathrm{GeV}\right)$. The inclusion of the NLL+NLO QED corrections reduces the scale variation band by roughly a factor 1.5-2. As in the Tevatron case the QED uncertainty is dominated by the renormalization (resummation) scale dependence at LL (NLL+NLO). 


\section{Summary}

In this paper we have extended the QCD transverse-momentum $\left(q_{T}\right)$ resummation formalism of refs. [46, 47] in order to deal with the simultaneous emission of QCD and QED initial state parton radiation. In particular, through the application of the Abelianization algorithm of refs. [56, 57], we have obtained analytic resummed results which are valid for the production of generic neutral and colourless high-mass systems in hadronic collisions.

We have applied our formalism to the transverse-momentum $\left(q_{T}\right)$ distribution of onshell $Z$ bosons produced at the Tevatron and the LHC. Starting from the known QCD results at next-to-next-to-leading logarithmic accuracy (NNLL) and next-to-next-to-leading order (NNLO) (i.e. $\mathcal{O}\left(\alpha_{S}^{2}\right)$ ) [58, 59], we have included the resummation of the logarithmically enhanced QED contributions at small values of $q_{T}$ up to next-to-leading logarithmic accuracy (NLL) and of the mixed QCD-QED contributions at leading logarithmic accuracy (LL). Our results have been consistently matched with the next-to-leading fixed-order (NLO) results (i.e. $\mathcal{O}\left(\alpha^{2}\right)$ ) in QED at small, intermediate and large values of $q_{T}$.

We have found that the resummation of the QED contributions at leading-logarithmic accuracy has the effect of slightly shifting (roughly $\mathcal{O}( \pm 1 \%)$ ) part of the cross section to higher values of $q_{T}$. The NLL+NLO QED effects at the central value of the scales are $\mathcal{O}(+1 \%)$ and rather flat for the entire $q_{T}$ region considered $\left(q_{T} \lesssim 40 \mathrm{GeV}\right)$.

We have then performed a study of the scale dependence of the QED contribution in order to estimate the corresponding perturbative uncertainty. We have shown that the scale variation band at LL is of $\mathcal{O}(2-4 \%)$ and that the inclusion of the NLL+NLO QED results reduces the scale variation band by roughly a factor 1.5-2. The QED uncertainty is dominated by the renormalization and resummation scale dependence at LL and NLL+NLO respectively.

In conclusion we have found that, given the present theoretical and experimental accuracy, the QED resummation effects for on-shell $Z$ boson production are not negligible. In particular the inclusion of the NLL+NLO QED corrections reduces the effect of the renormalization scale ambiguity of the QED coupling $\alpha$ by consistently including running coupling effects.

The inclusion of the QED effects from the leptonic decay of the $Z$ boson and the generalization of our results to the case of charged final states (e.g. $\left.W^{ \pm}\right)$are left for future investigations.

\section{Acknowledgments}

We gratefully acknowledge Stefano Catani, Daniel de Florian and Alessandro Vicini for useful discussions and comments on the manuscript. G.S. would like to thank also Germán Rodrigo and Félix Driencourt-Mangin for interesting discussions. This research was supported in part by Fondazione Cariplo under the grant number 2015-0761 and by the COST Action CA16201 PARTICLEFACE. 
Open Access. This article is distributed under the terms of the Creative Commons Attribution License (CC-BY 4.0), which permits any use, distribution and reproduction in any medium, provided the original author(s) and source are credited.

\section{References}

[1] S.D. Drell and T.-M. Yan, Massive Lepton Pair Production in Hadron-Hadron Collisions at High-Energies, Phys. Rev. Lett. 25 (1970) 316 [Erratum ibid. 25 (1970) 902] [INSPIRE].

[2] R. Hamberg, W.L. van Neerven and T. Matsuura, A complete calculation of the order $\alpha_{s}^{2}$ correction to the Drell-Yan K factor, Nucl. Phys. B 359 (1991) 343 [Erratum ibid. B 644 (2002) 403] [INSPIRE].

[3] R.V. Harlander and W.B. Kilgore, Next-to-next-to-leading order Higgs production at hadron colliders, Phys. Rev. Lett. 88 (2002) 201801 [hep-ph/0201206] [INSPIRE].

[4] C. Anastasiou, L.J. Dixon, K. Melnikov and F. Petriello, High precision QCD at hadron colliders: Electroweak gauge boson rapidity distributions at NNLO, Phys. Rev. D 69 (2004) 094008 [hep-ph/0312266] [INSPIRE].

[5] K. Melnikov and F. Petriello, The $W$ boson production cross section at the LHC through $O\left(\alpha_{s}^{2}\right)$, Phys. Rev. Lett. 96 (2006) 231803 [hep-ph/0603182] [INSPIRE].

[6] K. Melnikov and F. Petriello, Electroweak gauge boson production at hadron colliders through $O\left(\alpha_{s}^{2}\right)$, Phys. Rev. D 74 (2006) 114017 [hep-ph/0609070] [INSPIRE].

[7] R. Gavin, Y. Li, F. Petriello and S. Quackenbush, FEWZ 2.0: A code for hadronic Z production at next-to-next-to-leading order, Comput. Phys. Commun. 182 (2011) 2388 [arXiv: 1011.3540] [INSPIRE].

[8] S. Catani, L. Cieri, G. Ferrera, D. de Florian and M. Grazzini, Vector boson production at hadron colliders: a fully exclusive QCD calculation at NNLO,

Phys. Rev. Lett. 103 (2009) 082001 [arXiv:0903.2120] [INSPIRE].

[9] S. Catani, G. Ferrera and M. Grazzini, W Boson Production at Hadron Colliders: The Lepton Charge Asymmetry in NNLO QCD, JHEP 05 (2010) 006 [arXiv:1002.3115] [INSPIRE].

[10] R. Boughezal, C. Focke, X. Liu and F. Petriello, $W$-boson production in association with a jet at next-to-next-to-leading order in perturbative QCD, Phys. Rev. Lett. 115 (2015) 062002 [arXiv: 1504.02131] [INSPIRE].

[11] A. Gehrmann-De Ridder, T. Gehrmann, E.W.N. Glover, A. Huss and T.A. Morgan, Precise $Q C D$ predictions for the production of a $Z$ boson in association with a hadronic jet, Phys. Rev. Lett. 117 (2016) 022001 [arXiv:1507.02850] [INSPIRE].

[12] S. Dittmaier and M. Krämer, Electroweak radiative corrections to $W$ boson production at hadron colliders, Phys. Rev. D 65 (2002) 073007 [hep-ph/0109062] [INSPIRE].

[13] U. Baur and D. Wackeroth, Electroweak radiative corrections to $p \bar{p} \rightarrow W^{ \pm} \rightarrow \ell^{ \pm} \nu$ beyond the pole approximation, Phys. Rev. D 70 (2004) 073015 [hep-ph/0405191] [INSPIRE].

[14] V.A. Zykunov, Radiative corrections to the Drell-Yan process at large dilepton invariant masses, Phys. Atom. Nucl. 69 (2006) 1522 [InSPIRE]. 
[15] A. Arbuzov et al., One-loop corrections to the Drell-Yan process in SANC. I. The Charged current case, Eur. Phys. J. C 46 (2006) 407 [Erratum ibid. C 50 (2007) 505] [hep-ph/0506110] [INSPIRE].

[16] C.M. Carloni Calame, G. Montagna, O. Nicrosini and A. Vicini, Precision electroweak calculation of the charged current Drell-Yan process, JHEP 12 (2006) 016 [hep-ph/0609170] [INSPIRE].

[17] U. Baur, O. Brein, W. Hollik, C. Schappacher and D. Wackeroth, Electroweak radiative corrections to neutral current Drell-Yan processes at hadron colliders, Phys. Rev. D 65 (2002) 033007 [hep-ph/0108274] [INSPIRE].

[18] V.A. Zykunov, Weak radiative corrections to Drell-Yan process for large invariant mass of di-lepton pair, Phys. Rev. D 75 (2007) 073019 [hep-ph/0509315] [INSPIRE].

[19] C.M. Carloni Calame, G. Montagna, O. Nicrosini and A. Vicini, Precision electroweak calculation of the production of a high transverse-momentum lepton pair at hadron colliders, JHEP 10 (2007) 109 [arXiv:0710.1722] [INSPIRE].

[20] A. Arbuzov et al., One-loop corrections to the Drell-Yan process in SANC. (II). The Neutral current case, Eur. Phys. J. C 54 (2008) 451 [arXiv:0711.0625] [INSPIRE].

[21] L. Barze, G. Montagna, P. Nason, O. Nicrosini, F. Piccinini and A. Vicini, Neutral current Drell-Yan with combined QCD and electroweak corrections in the POWHEG BOX, Eur. Phys. J. C 73 (2013) 2474 [arXiv:1302.4606] [INSPIRE].

[22] A. Kotikov, J.H. Kuhn and O. Veretin, Two-Loop Formfactors in Theories with Mass Gap and Z-Boson Production, Nucl. Phys. B 788 (2008) 47 [hep-ph/0703013] [INSPIRE].

[23] W.B. Kilgore and C. Sturm, Two-Loop Virtual Corrections to Drell-Yan Production at order $\alpha_{s} \alpha^{3}$, Phys. Rev. D 85 (2012) 033005 [arXiv: 1107.4798] [InSPIRE].

[24] R. Bonciani, S. Di Vita, P. Mastrolia and U. Schubert, Two-Loop Master Integrals for the mixed EW-QCD virtual corrections to Drell-Yan scattering, JHEP 09 (2016) 091 [arXiv: 1604.08581] [INSPIRE].

[25] R. Bonciani, F. Buccioni, R. Mondini and A. Vicini, Double-real corrections at $\mathcal{O}\left(\alpha \alpha_{s}\right)$ to single gauge boson production, Eur. Phys. J. C 77 (2017) 187 [arXiv:1611.00645] [INSPIRE].

[26] S. Dittmaier, A. Huss and C. Schwinn, Mixed QCD-electroweak $O\left(\alpha_{s} \alpha\right)$ corrections to Drell-Yan processes in the resonance region: pole approximation and non-factorizable corrections, Nucl. Phys. B 885 (2014) 318 [arXiv:1403.3216] [INSPIRE].

[27] S. Dittmaier, A. Huss and C. Schwinn, Dominant mixed QCD-electroweak $O\left(\alpha_{s} \alpha\right)$ corrections to Drell-Yan processes in the resonance region, Nucl. Phys. B 904 (2016) 216 [arXiv: 1511.08016] [INSPIRE].

[28] C.M. Carloni Calame et al., Precision Measurement of the W-Boson Mass: Theoretical Contributions and Uncertainties, Phys. Rev. D 96 (2017) 093005 [arXiv:1612.02841] [INSPIRE].

[29] CDF, D0 collaborations, T.A. Aaltonen et al., Combination of CDF and D0 W-Boson Mass Measurements, Phys. Rev. D 88 (2013) 052018 [arXiv:1307.7627] [InSPIRE].

[30] ATLAS collaboration, Measurement of the $W$-boson mass in pp collisions at $\sqrt{s}=7 \mathrm{TeV}$ with the ATLAS detector, Eur. Phys. J. C 78 (2018) 110 [arXiv:1701.07240] [INSPIRE]. 
[31] R.K. Ellis, G. Martinelli and R. Petronzio, Lepton Pair Production at Large Transverse Momentum in Second Order QCD, Nucl. Phys. B 211 (1983) 106 [INSPIRE].

[32] P.B. Arnold and M.H. Reno, The Complete Computation of High $\mathrm{p}_{T} W$ and $Z$ Production in 2nd Order QCD, Nucl. Phys. B 319 (1989) 37 [Erratum ibid. B 330 (1990) 284] [inSPIRE].

[33] R.J. Gonsalves, J. Pawlowski and C.-F. Wai, QCD Radiative Corrections to Electroweak Boson Production at Large Transverse Momentum in Hadron Collisions, Phys. Rev. D 40 (1989) 2245 [inSPIRE].

[34] A. Gehrmann-De Ridder, T. Gehrmann, E.W.N. Glover, A. Huss and D.M. Walker, Next-to-Next-to-Leading-Order QCD Corrections to the Transverse Momentum Distribution of Weak Gauge Bosons, Phys. Rev. Lett. 120 (2018) 122001 [arXiv:1712.07543] [INSPIRE].

[35] Y.L. Dokshitzer, D. Diakonov and S.I. Troian, On the Transverse Momentum Distribution of Massive Lepton Pairs, Phys. Lett. B 79 (1978) 269 [INSPIRE].

[36] Y.L. Dokshitzer, D. Diakonov and S.I. Troian, Hard Processes in Quantum Chromodynamics, Phys. Rept. 58 (1980) 269 [INSPIRE].

[37] G. Parisi and R. Petronzio, Small Transverse Momentum Distributions in Hard Processes, Nucl. Phys. B 154 (1979) 427 [inSPIRE].

[38] G. Curci, M. Greco and Y. Srivastava, QCD Jets From Coherent States, Nucl. Phys. B 159 (1979) 451 [inSPIRE].

[39] J.C. Collins and D.E. Soper, Back-To-Back Jets in QCD, Nucl. Phys. B 193 (1981) 381 [Erratum ibid. B 213 (1983) 545] [INSPIRE].

[40] J.C. Collins and D.E. Soper, Back-To-Back Jets: Fourier Transform from B to K-Transverse, Nucl. Phys. B 197 (1982) 446 [InSPIRE].

[41] J.C. Collins, D.E. Soper and G.F. Sterman, Transverse Momentum Distribution in Drell-Yan Pair and $W$ and $Z$ Boson Production, Nucl. Phys. B 250 (1985) 199 [InSPIRE].

[42] J. Kodaira and L. Trentadue, Summing Soft Emission in QCD, Phys. Lett. B 112 (1982) 66 [INSPIRE].

[43] J. Kodaira and L. Trentadue, Soft gluon effects in perturbative quantum chromodynamics, SLAC-PUB-2934 (1982).

[44] J. Kodaira and L. Trentadue, Single Logarithm Effects in electron-Positron Annihilation, Phys. Lett. B 123 (1983) 335 [INSPIRE].

[45] D. de Florian and M. Grazzini, The Structure of large logarithmic corrections at small transverse momentum in hadronic collisions, Nucl. Phys. B 616 (2001) 247 [hep-ph/0108273] [INSPIRE].

[46] S. Catani, D. de Florian and M. Grazzini, Universality of nonleading logarithmic contributions in transverse momentum distributions, Nucl. Phys. B 596 (2001) 299 [hep-ph/0008184] [INSPIRE].

[47] G. Bozzi, S. Catani, D. de Florian and M. Grazzini, Transverse-momentum resummation and the spectrum of the Higgs boson at the LHC, Nucl. Phys. B 737 (2006) 73 [hep-ph/0508068] [INSPIRE].

[48] S. Catani and M. Grazzini, QCD transverse-momentum resummation in gluon fusion processes, Nucl. Phys. B 845 (2011) 297 [arXiv: 1011.3918] [INSPIRE]. 
[49] S. Catani, L. Cieri, D. de Florian, G. Ferrera and M. Grazzini, Universality of transverse-momentum resummation and hard factors at the $N N L O$,

Nucl. Phys. B 881 (2014) 414 [arXiv:1311.1654] [INSPIRE].

[50] V.V. Sudakov, Vertex parts at very high-energies in quantum electrodynamics, Sov. Phys. JETP 3 (1956) 65 [INSPIRE].

[51] S. Alioli et al., Precision studies of observables in $p p \rightarrow W \rightarrow l \nu_{l}$ and $p p \rightarrow \gamma, Z \rightarrow l^{+} l^{-}$ processes at the LHC, Eur. Phys. J. C 77 (2017) 280 [arXiv:1606.02330] [InSPIRE].

[52] A. Gehrmann-De Ridder, T. Gehrmann, E.W.N. Glover, A. Huss and T.A. Morgan, NNLO QCD corrections for Drell-Yan $p_{T}^{Z}$ and $\phi^{*}$ observables at the LHC, JHEP 11 (2016) 094 [arXiv: 1610.01843] [INSPIRE].

[53] W. Bizoń et al., Fiducial distributions in Higgs and Drell-Yan production at $N^{3} L L+N N L O$, arXiv: 1805.05916 [INSPIRE].

[54] D. de Florian, M. Der and I. Fabre, $Q C D \oplus Q E D$ NNLO corrections to Drell-Yan production, arXiv: 1805.12214 [INSPIRE].

[55] S. Catani, E. D'Emilio and L. Trentadue, The Gluon Form-factor to Higher Orders: Gluon Gluon Annihilation at Small Q- transverse, Phys. Lett. B 211 (1988) 335 [INSPIRE].

[56] D. de Florian, G.F.R. Sborlini and G. Rodrigo, QED corrections to the Altarelli-Parisi splitting functions, Eur. Phys. J. C 76 (2016) 282 [arXiv:1512.00612] [INSPIRE].

[57] D. de Florian, G.F.R. Sborlini and G. Rodrigo, Two-loop QED corrections to the Altarelli-Parisi splitting functions, JHEP 10 (2016) 056 [arXiv:1606.02887] [INSPIRE].

[58] G. Bozzi, S. Catani, G. Ferrera, D. de Florian and M. Grazzini, Transverse-momentum resummation: A Perturbative study of $Z$ production at the Tevatron, Nucl. Phys. B 815 (2009) 174 [arXiv:0812.2862] [InSPIRE].

[59] G. Bozzi, S. Catani, G. Ferrera, D. de Florian and M. Grazzini, Production of Drell-Yan lepton pairs in hadron collisions: Transverse-momentum resummation at next-to-next-to-leading logarithmic accuracy, Phys. Lett. B 696 (2011) 207 [arXiv: 1007.2351] [INSPIRE].

[60] Particle Data Group collaboration, C. Patrignani et al., Review of Particle Physics, Chin. Phys. C 40 (2016) 100001 [InSPIRE].

[61] NNPDF collaboration, R.D. Ball et al., Parton distributions from high-precision collider data, Eur. Phys. J. C 77 (2017) 663 [arXiv:1706.00428] [INSPIRE].

[62] NNPDF collaboration, V. Bertone et al., Illuminating the photon content of the proton within a global PDF analysis, SciPost Phys. 5 (2018) 008 [arXiv:1712.07053] [INSPIRE].

[63] A. Manohar, P. Nason, G.P. Salam and G. Zanderighi, How bright is the proton? A precise determination of the photon parton distribution function, Phys. Rev. Lett. 117 (2016) 242002 [arXiv: 1607.04266] [INSPIRE].

[64] A.V. Manohar, P. Nason, G.P. Salam and G. Zanderighi, The Photon Content of the Proton, JHEP 12 (2017) 046 [arXiv:1708.01256] [INSPIRE].

[65] S. Catani, D. de Florian, G. Ferrera and M. Grazzini, Vector boson production at hadron colliders: transverse-momentum resummation and leptonic decay, JHEP 12 (2015) 047 [arXiv: 1507.06937] [INSPIRE]. 